\title{
Fictitious Parts of the Load in Thermoelasticity
}

\author{
Janusz Jankowski \\ Poznan University of Technology, Institute of Applied Mechanics \\ ul. Piotrowo 3, 60-965 Poznań, Poland \\ janusz.jankowski@put.poznan.pl
}

(Received: 14 May 2008; published online: 20 November 2008)

\begin{abstract}
The three-dimensional coupled quasi-static problem of linear thermoelasticity is presented. The concept is based on a spatial extension of a region occupied by the considered body and on spatial formulation of a new fictitious load. All the outside objects are termed here fictitious ones. The solution of the initial-space value problem includes fictitious displacement-temperature components. Capacity values of approximate fictitious components are calculated from a boundary condition contracted to the finite time interval. The approximate solution to the primary thermoelastic problem is obtained by contracting in space the approximate solution to the initial-space value problem. It enables us to determine the thermoelastic flow.
\end{abstract}

Key words: coupled thermoelasticity, fictitious components, convolution products

\section{INTRODUCTION}

We consider the initial-boundary value problem of the quasi-static coupled thermoelasticity. At first a bounded region occupied by the model of the body is extending over the space in which the initial-space value problem is defined, thus corresponding with the primary initialboundary value problem. The representation of displacement and body force vectors by means of the Helmholtz potentials led to the solution to the initial-space value problem. For some verification of the boundary condition a proposal is made to replace multidimensional integrals of the outside parts of the load with functions called fictitious components. "Fictitious" is a common term used for some ideas that do not concern the region really occupied by the model of the body. The fictitious components are determined in an approximate way in order to satisfy the boundary condition with arbitrary accuracy, at the same time, exactly satisfying the governing equations and the initial condition. Capacity values of the approximate temperature fictitious components located in some fictitious places (beyond the body region) are calculated in fictitious time defined as a discrete set of fictitious instants. Fictitious instants appear in temperature fictitious components only. To obtain an approximate solution a finite time interval is introduced in which the boundary condition is verified. The test instants divide the finite time into sec- tions and constitute a discrete set called test time. Capacity values of the displacement fictitious components are time functions and therefore an algorithm designed for their determination concerns the current instant. An approximate solution is obtained by mathematical contraction to the body region of the solution to the initial-space value problem with the derived capacities of temperaturedisplacement fictitious components. It means that the stress tensor and heat flux vector should be determined as appropriate derivatives of the solution to the thermoelastic problem.

The coupled initial condition includes the initial change of dilatation. It was proposed as a consequence of the coupled current states constituting the thermoelastic process. In particular the initial condition as a description of the global initial state also has to be coupled.

\section{THERMOELASTIC PROBLEM}

Let the homogeneous and isotropic linearly-elastic body occupy the region $D$ in three-dimensional Euclidean space $Z$. Let vector $v_{i}$ denote the outward normal to the boundary $\partial D$. Assuming that the effect of the inertia may be omitted, the thermoelastic problem consists of the governing relations 


$$
\begin{gathered}
\mu \cdot u_{i, j j}(\mathbf{x}, t)+(\lambda+\mu) \cdot u_{j, i j}(\mathbf{x}, t)-\gamma \cdot T_{, i}(\mathbf{x}, t)=-X_{i}(\mathbf{x}, t), \\
T_{, j j}(\mathbf{x}, t)-\frac{\partial}{\partial t}\left[\frac{1}{\kappa} \cdot T(\mathbf{x}, t)+\eta \cdot u_{j, j}(\mathbf{x}, t)\right]=-\frac{1}{{ }_{o} \lambda} \cdot W(\mathbf{x}, t), \\
\mathbf{x} \in D, t>0
\end{gathered}
$$

initial condition

$$
\begin{gathered}
T(\mathbf{x}, 0) / \kappa+\eta \cdot u_{j, j}(\mathbf{x}, 0)=g(\mathbf{x}), \\
\mathbf{x} \in D
\end{gathered}
$$

and boundary conditions

$$
\begin{gathered}
\mu \cdot\left[u_{i, j}\left({ }_{1} \mathbf{r}, t\right)+u_{j, i}\left({ }_{1} \mathbf{r}, t\right)\right] \cdot v_{j}\left({ }_{1} \mathbf{r}, t\right)+ \\
+\left[\lambda \cdot u_{k, k}\left({ }_{1} \mathbf{r}, t\right)-\gamma \cdot T\left({ }_{1} \mathbf{r}, t\right)\right] \cdot v_{i}\left({ }_{1} \mathbf{r}, t\right)={ }^{v} P_{i}\left({ }_{1} \mathbf{r}, t\right), \\
u_{i}\left({ }_{2} \mathbf{r}, t\right)=U_{i}\left({ }_{2} \mathbf{r}, t\right), \\
{ }_{o} \lambda \cdot T_{, j}(\mathbf{r}, t) \cdot v_{j}(\mathbf{r}, t)+\alpha \cdot T(\mathbf{r}, t)= \\
=V(\mathbf{r}, t)+\alpha \cdot{ }_{b} T(\mathbf{r}, t), \quad t>0, \\
\mathbf{r} \in \partial D,{ }_{1} \mathbf{r} \in \partial{ }_{1} D,{ }_{2} \mathbf{r} \in \partial_{2} D, \quad \partial_{1} D \cup \partial{ }_{2} D=\partial D, \\
\partial{ }_{1} D \cap \partial{ }_{2} D=\Theta .
\end{gathered}
$$

Here $u_{i}$ is the displacement vector, $T-$ the temperature, $X_{i}$ - the components of body force, $W$ - heat generated per unit volume and time, $\mu, \lambda$ - Lamé constants, $\gamma-$ thermal expansion, $\kappa-$ thermal diffusivity, and $\eta-$ coefficient of thermomechanical coupling. Symbol $g$ stands here for the thermoelastic initial state, ${ }^{v} P_{i}-$ the surface traction on $\partial_{1} D, U_{i}$ - the displacement on $\partial_{2} D$, $V$ - the heat generated per unit surface and time, $\alpha-$ coefficient of surface heat transfer, and ${ }_{b} T$ - the surrounding temperature. In the (1-3) system of equations a local thermoelastic state (which is to be determined) arises at the left-hand side of the equality signs. Therefore we will call the state the unknown. The unknown is subjected to some mathematical operations called the operator. On the other hand, the right-hand sides of the equations will be called the (thermoelastic) load.

Thermoelastic flow through a surface with the normal $v_{i}$ is defined by the expressions:

$$
\begin{aligned}
{ }^{v} P_{i}(\mathbf{x}, t) & \equiv \sigma_{j i}(\mathbf{x}, t) \cdot v_{j}(\mathbf{x}, t), \\
\mathbf{x} & \in D, \quad t>0, \\
{ }^{v} q(\mathbf{x}, t) & \equiv q_{j}(\mathbf{x}, t) \cdot v_{j}(\mathbf{x}, t), \\
\mathbf{x} & \in D, \quad t>0 .
\end{aligned}
$$

The Cauchy formula (4) defines the mechanical part of the flow called surface traction ${ }^{v} P_{i}$, while the thermal part (5) is defined by the surface heat flow ${ }^{v} q$. The thermo- elastic stress tensor is defined by the Duhamel-Neumann equation

$$
\sigma_{i j}=\mu \cdot\left(u_{i, j}+u_{j, i}\right)+\left(\lambda \cdot u_{k, k}-\gamma \cdot T\right) \cdot \delta_{i j},
$$

and the heat flux vector by the Fourier law

$$
q_{i}=-{ }_{o} \lambda \cdot T_{, i}
$$

where ${ }_{o} \lambda=\kappa \cdot{ }_{\varepsilon} c$ thermal conductivity, ${ }_{\varepsilon} c-$ specific heat at constant strain, and $\delta_{i j}$ - the Kronecker Delta.

We will call an interval $(0, \infty)$ the time, $(0, t)$ the current time, while $(0, \tilde{t})$ the finite time, where $\tilde{t}>t$ is the final instant and $t>0$ is the current instant.

The governing equations (1) may describe the phenomena by means of the displacement-temperature pair $\langle u \mid T\rangle$, provided that the thermoelastic boundary and the initial load is taken into account. This will lead to the initial-boundary value problem as a model of the thermomechanical process. For a fixed current instant, set $\{\langle u \mid T\rangle(\mathbf{x}, t), \mathbf{x} \in D\}$ is called a global thermoelastic state, while value $\langle u \mid T\rangle(\mathbf{x}, t)-$ a local one.

\section{INITIAL-SPACE VALUE PROBLEM}

We will seek a solution of the thermoelastic problem in an approximate way in the class of twice differentiable functions. Let the fictitious model occupy space $Z$. For the model of the process taking place in the Cartesian product $Z \times(0, \infty)$, a continuous and bounded in infinity load is defined as a model of interactions on the body

$$
\begin{gathered}
\bar{g}: Z \rightarrow R, \lim _{\mathbf{z} \rightarrow \infty}|\bar{g}(\mathbf{z})|<\infty, \\
\bar{W}: Z \times(0, \infty) \rightarrow R, \quad \lim _{\mathbf{z} \rightarrow \infty}|\bar{W}(\mathbf{z}, t)|<\infty, \\
\bar{X}_{i}: Z \times(0, \infty) \rightarrow R, \\
\lim _{\mathbf{z} \rightarrow \infty}\left|\bar{X}_{i}(\mathbf{z}, t)\right|<\infty, \quad \mathbf{z} \in Z, t>0
\end{gathered}
$$

and both temperature and displacement fields are defined as well

$$
\begin{aligned}
& \bar{T}: Z \times(0, \infty) \rightarrow R, \\
& \bar{u}_{i}: Z \times(0, \infty) \rightarrow R .
\end{aligned}
$$

We assume that a new load does not differ within region $D$ from the thermoelastic load existing there

$$
\begin{aligned}
& \bar{g}(\mathbf{z})=g(\mathbf{z}), \quad \bar{W}(\mathbf{z}, t)=W(\mathbf{z}, t), \\
& \bar{X}_{i}(\mathbf{z}, t)=X_{i}(\mathbf{z}, t), \quad \mathbf{z} \in D, t>0 .
\end{aligned}
$$


For the thermoelastic process taking place in the Cartesian product $Z \times(0, \infty)$, the initial-space value problem contains improper boundary conditions

$$
\begin{gathered}
\mu \cdot \bar{u}_{i, j j}(\mathbf{z}, t)+(\lambda+\mu) \cdot \bar{u}_{j, i j}(\mathbf{z}, t)-\gamma \cdot \bar{T}_{, i}(\mathbf{z}, t)= \\
=-\bar{X}_{i}(\mathbf{z}, t), \\
\bar{T}_{, j j}(\mathbf{z}, t)-\frac{\partial}{\partial t}\left[\frac{1}{\kappa} \cdot \bar{T}(\mathbf{z}, t)+\eta \cdot \bar{u}_{j, j}(\mathbf{z}, t)\right]= \\
=-\frac{1}{\lambda} \cdot \bar{W}(\mathbf{z}, t), \\
\quad \mathbf{z} \in Z, \quad t>0, \\
\bar{T}(\mathbf{z}, 0) / \kappa+\eta \cdot \bar{u}_{j, j}(\mathbf{z}, 0)=\bar{g}(\mathbf{z}), \quad \mathbf{z} \in Z \\
\lim _{\mathbf{z} \rightarrow \infty}|\bar{T}(\mathbf{z}, t)|<\infty, \quad \lim _{\mathbf{z} \rightarrow \infty}\left|\bar{u}_{i}(\mathbf{z}, t)\right|<\infty, \quad t>0 .
\end{gathered}
$$

Suppose $\mathbf{F}$ is a vector point function with $r^{2} \cdot|\mathbf{F}|$ bounded at infinity $[1$, p. 326$]$. We assume that vectors $\bar{u}_{i}$ and $\bar{X}_{i}$ satisfy such a condition and we express them in terms of Helmholtz potentials

$$
\begin{gathered}
\bar{u}_{i}=\tilde{\lambda} \cdot \phi_{, i}+\tilde{\mu} \cdot \varepsilon_{i j k} \cdot \psi_{k, j}, \quad \psi_{k, k}=0, \\
\tilde{\lambda}=(\lambda+2 \cdot \mu)^{-1}, \tilde{\mu}=(\mu)^{-1} \\
\bar{X}_{i}=\beta_{, i}+\varepsilon_{i j k} \cdot \chi_{k, j}, \quad \chi_{k, k}=0 .
\end{gathered}
$$

$\varepsilon_{i j k}$ denotes here the permutation symbol, and zeroing of the divergence is called a marking condition. In an infinite thermoelastic space the Helmholtz potentials can be represented by the body force [2, p. 182]

$$
\begin{gathered}
\beta(\mathbf{z}, t)=-\frac{1}{4 \cdot \pi} \cdot \int_{Z} \frac{\bar{X}_{i, i}(\mathbf{z}, t)}{|\mathbf{z}-\mathbf{y}|} \cdot d \mathbf{y}+{ }_{o} \beta(t), \\
\chi_{i}(\mathbf{z}, t)=-\frac{1}{4 \cdot \pi} \cdot \int_{Z} \frac{\varepsilon_{i j k} \cdot \bar{X}_{k, j}(\mathbf{y}, t)}{|\mathbf{z}-\mathbf{y}|} \cdot d \mathbf{y}+{ }_{o} \chi_{i}(t), \\
\mathbf{z} \in Z, t>0 .
\end{gathered}
$$

For the problem (11-12) such temperatures and potentials that satisfy the following Helmholtz space problem are required:

$$
\begin{gathered}
{\left[\phi_{, j j}(\mathbf{z}, t)+\beta_{, i}(\mathbf{z}, t)-\gamma \cdot \bar{T}(\mathbf{z}, t)\right]_{, i}+} \\
+\varepsilon_{i j k} \cdot\left[\psi_{k, l l j}(\mathbf{z}, t)+\chi_{k, j}(\mathbf{z}, t)\right]=0, \\
\bar{T}_{, j j}(\mathbf{z}, t)-\frac{\partial}{\partial t}\left[\frac{1}{\kappa} \cdot \bar{T}(\mathbf{z}, t)+\eta \cdot \tilde{\lambda} \cdot \phi_{, j j}(\mathbf{z}, t)\right]= \\
=-\frac{1}{{ }_{o} \lambda} \cdot \bar{W}(\mathbf{z}, t), \quad \mathbf{z} \in Z, \quad t>0,
\end{gathered}
$$

$$
\begin{gathered}
\bar{T}(\mathbf{z}, 0) / \kappa+\eta \cdot \bar{u}_{j, j}(\mathbf{z}, 0)=\bar{g}(\mathbf{z}), \quad \mathbf{z} \in Z \\
\lim _{\mathbf{z} \rightarrow \infty}|\bar{T}(\mathbf{z}, t)|<\infty, \quad \lim _{\mathbf{z} \rightarrow \infty}\left|\bar{u}_{i}(\mathbf{z}, t)\right|<\infty, \quad t>0 .
\end{gathered}
$$

For the Helmholtz potentials we have

$$
\begin{gathered}
\phi(\mathbf{z}, t)=[\gamma \cdot \bar{T}(Z, t)-\beta(Z, t)] * E(\mathbf{z}), \\
\psi_{i}(\mathbf{z}, t)=-\chi_{i}(Z, t) * E(\mathbf{z}) .
\end{gathered}
$$

We can check that the functions satisfying the problem (11-12) have the form [5]:

$$
\begin{gathered}
\bar{T}(\mathbf{z}, t)= \\
=\tilde{\kappa} \cdot\left[\eta \cdot \tilde{\lambda} \cdot \frac{\partial}{\partial t} \beta(Z \times(0, t))+\bar{W}(Z \times(0, t)) /{ }_{o} \lambda\right] * F(\mathbf{z}, t)+ \\
+\tilde{\kappa} \cdot[\bar{g}(Z)+\eta \cdot \tilde{\lambda} \cdot \beta(Z, 0)] * F(\mathbf{z}, t), \\
\mathbf{z} \in Z, t>0, \\
\bar{u}_{i}(\mathbf{z}, t)= \\
=\tilde{\lambda} \cdot[\gamma \cdot \bar{T}(Z, t)-\beta(Z, t)] * E_{, i}(\mathbf{z})+ \\
-\tilde{\mu} \cdot \varepsilon_{i j k} \cdot \chi_{k}(Z, t) * E_{, j}(\mathbf{z}) .
\end{gathered}
$$
ity

Here $\tilde{\kappa}$ plays the role of the substitute thermal diffusiv-

$$
\frac{1}{\tilde{\kappa}}=\frac{1}{\kappa}+\eta \cdot \gamma \cdot{ }_{o} \lambda
$$

The denotation $(Z, \cdot) *$ will be called the Laplace convolution multiplication on set $Z$, and its result - the Laplace convolution product (of two functions)

$$
\begin{gathered}
f(Z, t) * E(\mathbf{z}) \equiv \int_{Z} f(\mathbf{y}, t) \cdot E(\mathbf{z}-\mathbf{y}) \cdot d \mathbf{y}, \\
\mathbf{z} \in Z .
\end{gathered}
$$

Function $E$ is the fundamental solution of the elliptic operator

$$
E(\mathbf{z}) \equiv-(4 \cdot \pi \cdot|\mathbf{z}|)^{-1}, \quad \mathbf{z} \neq 0
$$

The denotation $(Z) *$ will be called the space convolution multiplication on set $Z$, and its result the space convolution product

$$
\begin{gathered}
f(Z) * F(\mathbf{z}, t) \equiv \int_{Z} f(\mathbf{y}) \cdot F(\mathbf{z}-\mathbf{y}, t) \cdot d \mathbf{y}, \\
\mathbf{z} \in Z, \quad t>0 .
\end{gathered}
$$

Function $F$ is the fundamental solution of the parabolic operator 


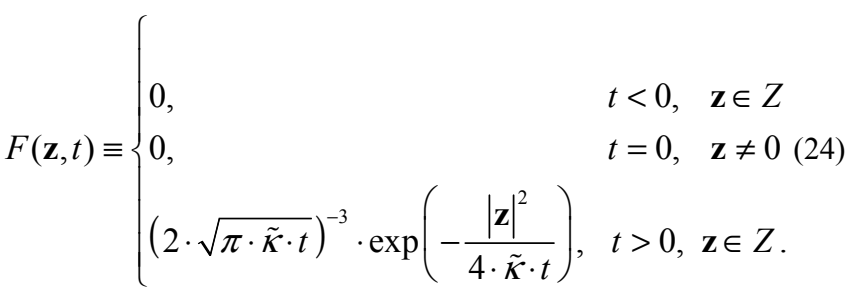

The denotation $(Z \times(0, t)) *$ will be called the spacetime convolution multiplication on set $Z \times(0, t)$, and its result - the space-time convolution product

$$
\begin{gathered}
f(Z \times(0, t)) * F(\mathbf{z}, t) \equiv \\
\equiv \int_{0}^{t} \int_{Z} f(\mathbf{y}, s) \cdot F(\mathbf{z}-\mathbf{y}, t-s) \cdot d \mathbf{y} d s, \\
\mathbf{z} \in Z, \quad t>0 .
\end{gathered}
$$

The space-time convolution satisfies an important identity for any integrable function $f[3$, p. 230]

$$
\begin{gathered}
\frac{\partial}{\partial t}[f(Z \times(0, t)) * F(\mathbf{z}, t)]-\tilde{\kappa} \cdot[f(Z \times(0, t)) * F(\mathbf{z}, t)]_{, j j}= \\
=f(\mathbf{z}, t), \quad \mathbf{z} \in Z, t>0 .
\end{gathered}
$$

An improper boundary condition for function $\bar{T}$ is met due to the properties of the $F$ function and boundedness (8) in infinity. Functions convolutionally multiplied by $F$ are assumed in a continuous and bounded manner, hence the formula (18) presents a continuous and bounded function in $Z \times(0, \tau)$, where $0<\tau<\infty$ [4, pp. 361-363]. So function $\bar{T}$ is bounded in infinity and from relation (14) it appears that the Laplace convolutions in formula (19) gives a continuously differentiable function on a bounded and closed domain, if the convolution is defined on this domain [4, p. 191]. Therefore, improper boundary conditions for functions $u_{i}$ are also satisfied.

\section{TEMPERATURE-DISPLACEMENT FICTITIOUS COMPONENTS}

Let us make use of the law of additivity of the integral as the set function, and make use of the formula (10)

$$
\begin{gathered}
\bar{T}(\mathrm{z}, t)=\tilde{\kappa} \cdot\left[\eta \cdot \tilde{\lambda} \cdot \frac{\partial}{\partial t} \beta(D \times(0, t))+\right. \\
\left.+\frac{1}{{ }_{o} \lambda} \cdot W(D \times(0, t))\right] * F(\mathbf{z}, t)+ \\
+\tilde{\kappa} \cdot[g(D)+\eta \cdot \tilde{\lambda} \cdot \beta(D, 0)] * F(\mathbf{z}, t)+ \\
+{ }_{f} T(\mathbf{z}, t), \quad \mathbf{z} \in Z, \quad t>0,
\end{gathered}
$$

$$
\begin{aligned}
\bar{u}_{i}(\mathbf{z}, t)= & \tilde{\lambda} \cdot[\gamma \cdot \bar{T}(D, t)-\beta(D, t)] * E_{, i}(\mathbf{z})+ \\
& -\tilde{\mu} \cdot \varepsilon_{i j k} \cdot \chi_{k}(D, t) * E_{, j}(\mathbf{z})+ \\
& +{ }_{f} u_{i}(\mathbf{z}, t), \quad \mathbf{z} \in Z, t>0
\end{aligned}
$$

where the following denotations have been used

$$
\begin{gathered}
{ }_{f} u_{i}(\mathbf{z}, t) \equiv \tilde{\lambda} \cdot[\gamma \cdot \bar{T}(Z \backslash D, t)-\beta(Z \backslash D, t)] * E_{, i}(\mathbf{z})+ \\
-\varepsilon_{i j k} \cdot \chi_{k}(Z \backslash D, t) * E_{, j}(\mathbf{z}) / \mu, \\
{ }_{f} T(\mathbf{z}, t) \equiv \tilde{\kappa} \cdot\left[\eta \cdot \tilde{\lambda} \cdot \frac{\partial}{\partial t} \beta((Z \backslash D) \times(0, t))+\right. \\
\left.+\frac{1}{{ }_{o} \lambda} \cdot \bar{W}((Z \backslash D) \times(0, t))\right] * F(\mathbf{z}, t)+ \\
+\tilde{\kappa} \cdot[\eta \cdot \tilde{\lambda} \cdot \beta(Z \backslash D, 0)+\bar{g}(Z \backslash D)] * F(\mathbf{z}, t), \\
\mathbf{z} \in Z, t>0 .
\end{gathered}
$$

Functions ${ }_{f} T$ and ${ }_{f} u_{i}$ are the temperature and displacement fictitious components. They are defined in time $(0, \infty)$ on the whole space $Z$. The functions (27-28) are called the component integral because they satisfy strictly (11-12) and contain the fictitious components. The fictitious components have to satisfy the component admissibility conditions

$$
\begin{gathered}
\mu \cdot{ }_{f} u_{i, j j}(\mathbf{x}, t)+(\lambda+\mu) \cdot{ }_{f} u_{j, i j}(\mathbf{x}, t)=0, \\
{ }_{f} T_{, j j}(\mathbf{x}, t)=\frac{1}{\tilde{\kappa}} \cdot \frac{\partial}{\partial t}{ }_{f} T(\mathbf{x}, t), \\
\mathbf{x} \in D, \quad t>0, \\
T(\mathbf{x}, 0)=0, \quad \mathbf{x} \in D .
\end{gathered}
$$

It is easy to notice that assuming the displacement fictitious component in the form of a gradient of the elliptic fundamental solution and the temperature fictitious component in the form of the parabolic fundamental solution, the component admissibility conditions are fulfilled. Therefore, the approximate fictitious components will be searched for in the form:

$$
\begin{gathered}
{ }_{f}^{a} T(\mathbf{z}, t) \equiv \sum_{l=1}^{p} \sum_{m=1}^{n} T_{l}^{m} \cdot F\left(\mathbf{z}-{ }_{m} \mathbf{y}, t-{ }^{l} s\right), \\
t>0, \mathbf{z} \in Z, \quad T_{l}^{m},{ }^{l} s \in R, \quad{ }_{m} \mathbf{y} \in Z \backslash D, \\
{ }_{f}^{a} u_{i}(\mathbf{z}, t) \equiv \sum_{m=1}^{n} u_{j}^{m}(t) \cdot E_{, i j}\left(\mathbf{z}-{ }_{m} \mathbf{y}\right), \\
t>0, \mathbf{z} \in Z, \quad u_{i}^{m}(t) \in R, \\
u_{i}^{m}(0)=0, \quad{ }_{m} \mathbf{y} \in Z \backslash D .
\end{gathered}
$$


The functions (32-33) are called approximate fictitious components and the coefficients accompanying the fundamental solutions - component capacities: ${ }_{f}^{a} T$ - the approximate temperature component, and ${ }_{f}^{a} u_{k}-$ the approximate displacement component. Here ${ }_{m} \mathbf{y}$ is the fictitious place, ${ }^{l} s$ - the fictitious instant, $w_{l}^{m}, u_{k}^{m}(t)$ - the temperature-displacement capacity in the ${ }_{m} \mathbf{y}$ place, the sets $\left\{_{m} \mathbf{y}: m=\overline{1, n}\right\}$. $\left\{{ }^{l} s: l=\overline{1, p}\right\}$ fictitious location and time. The approximate fictitious components defined this way satisfy the component admissibility conditions.

Let us replace the fictitious components in the component integral with their approximate form (32-33)

$$
\begin{gathered}
\hat{T}(\mathbf{z}, t) \equiv \\
\equiv \tilde{\kappa} \cdot\left[\eta \cdot \tilde{\lambda} \cdot \frac{\partial}{\partial t} \beta(D \times(0, t))+\frac{1}{{ }_{o}} \cdot W(D \times(0, t))\right] * F(\mathbf{z}, t) \\
+\tilde{\kappa} \cdot[g(D)+\eta \cdot \tilde{\lambda} \cdot \beta(D, 0)] * F(\mathbf{z}, t)+{ }_{f}^{a} W(\mathbf{z}, t) \\
\mathbf{z} \in Z, \quad t>0 \\
\hat{u}_{i}(\mathbf{z}, t) \equiv \tilde{\lambda} \cdot[\gamma \cdot \hat{T}(D, t)-\beta(D, t)] * E_{, i}(\mathbf{z})+ \\
-\tilde{\mu} \cdot \varepsilon_{i j k} \cdot \chi_{k}(D, t) * E_{, j}(\mathbf{z})+ \\
+{ }_{f}^{a} u_{i}(\mathbf{z}, t) \\
\mathbf{z} \in Z, t>0 .
\end{gathered}
$$

These functions are the approximate integral. The capacities of the approximate components should be derived to satisfy the boundary condition (3) in the best possible way. Therefore, the time $(0, \infty)$ will be contracted to the finite time $(0, \tilde{t})$. The temperature capacity values can be determined independently. For the thermoelastic stress tensor (6) in the finite time the normal to the boundary surface will be assumed as independent of the current instant

$$
\begin{gathered}
2 \cdot \mu \cdot v_{j}\left({ }_{1} \mathbf{r}\right) \cdot \sum_{m=1}^{n} u_{k}^{m}(t) \cdot E_{, j j k}\left({ }_{1} \mathbf{r}, t\right)=\hat{P}_{i}\left({ }_{1} \mathbf{r}, t\right), \\
\mathbf{r} \in \partial_{1} D, t \in(0, \tilde{t}), \\
\sum_{m=1}^{n} u_{j}^{m}(t) \cdot E_{, i j}\left({ }_{2} \mathbf{r}-{ }_{m} \mathbf{y}\right)=\hat{U}_{i}\left({ }_{2} \mathbf{r}, t\right), \\
{ }_{2} \mathbf{r} \in \partial_{2} D, t \in(0, \tilde{t}), \\
\sum_{l=1}^{p} \sum_{m=1}^{n} w_{l}^{m} \cdot\left[{ }_{o} \lambda \cdot v_{i}(\mathbf{r}) \cdot F_{, i}+\alpha \cdot F\right]\left(\mathbf{r}-{ }_{m} \mathbf{y}, t-{ }^{l} s\right)=\hat{V}(\mathbf{r}, t), \\
\mathbf{r} \in \partial D, \quad t \in(0, \tilde{t})
\end{gathered}
$$

where the following denotations have been used

$$
\begin{gathered}
\hat{P}_{i}(\mathbf{r}, t) \equiv{ }^{v} P_{i}(\mathbf{r}, t)+ \\
+\tilde{\lambda} \cdot[\lambda \cdot \beta(\mathbf{r}, t)+2 \cdot \mu \cdot \gamma \cdot \hat{T}(\mathbf{r}, t)] \cdot v_{i}(\mathbf{r})+v_{j}(\mathbf{r}) \\
\cdot\left\{2 \cdot \mu \cdot \tilde{\lambda} \cdot[\beta(D, t)-\gamma \cdot \hat{T}(D, t)] * E_{, i j}(\mathbf{r})+\right. \\
\left.+\chi_{l}(D, t) *\left[\varepsilon_{i k l} \cdot E_{, k j}(\mathbf{r})+\varepsilon_{j k l} \cdot E_{, k i}(\mathbf{r})\right]\right\}, \\
\hat{U}_{i}(\mathbf{r}, t) \equiv U_{i}(\mathbf{r}, t)+\tilde{\lambda} \cdot[\beta(D, t)-\gamma \cdot \hat{T}(D, t)] * E_{, i}(\mathbf{r})+ \\
+\varepsilon_{i j k} \cdot \tilde{\mu} \cdot \chi_{k}(D, t) * E_{, j}(\mathbf{r}), \\
\hat{V}(\mathbf{r}, t) \equiv V(\mathbf{r}, t)+\alpha \cdot{ }_{b} T(\mathbf{r}, t)+ \\
-\tilde{\kappa} \cdot\left[\eta \cdot \tilde{\lambda} \cdot \frac{\partial}{\partial t} \beta(D \times(0, t))+\right. \\
\left.+\frac{1}{{ }_{o} \lambda} \cdot W(D \times(0, t))\right] *\left[v_{j}(\mathbf{r}) \cdot{ }_{o} \lambda \cdot F_{, j}(\mathbf{r}, t)+\alpha \cdot F(\mathbf{r}, t)\right]+ \\
-\tilde{\kappa} \cdot[g(D)+\eta \cdot \tilde{\lambda} \cdot \beta(D, 0)] *\left[v_{j}(\mathbf{r}) \cdot{ }_{o} \lambda \cdot F_{, j}(\mathbf{r}, t)+\alpha \cdot F(\mathbf{r}, t)\right] .
\end{gathered}
$$

We shall cover boundary $\partial D$ with mesh ${ }_{h} \mathbf{r}, h=\overline{1, N}$, where $N>n$. Let us call set $\left\{{ }^{l} t: l=\overline{1, p}\right\}$ of test instants ${ }^{k} t$ the test time, where $0<{ }^{1} t<{ }^{2} t<\ldots<{ }^{p} t<\tilde{t}$. We shall write the formula (38) at the nodes ${ }_{h} \mathbf{r} \in \partial D$ and the test instants ${ }^{k} t \in(0, \tilde{t})$

$$
\begin{gathered}
\sum_{l=1}^{p} \sum_{m=1}^{n} T_{l}^{m} \cdot B_{h m}^{k l}=\hat{V}\left({ }_{h} \mathbf{r},{ }^{k} t\right), \quad{ }_{h} \mathbf{r} \in \partial D, \\
h=\overline{1, N}, \quad{ }^{k} t \in(0, \tilde{t}), \quad k=\overline{1, p} . \\
B_{h m}^{k l} \equiv{ }_{o} \lambda \cdot F_{, j}\left({ }_{h} \mathbf{r}-{ }_{m} \mathbf{y},{ }^{k} t-{ }^{l} s\right) \cdot v_{j}\left({ }_{h} \mathbf{r}\right)+ \\
+\alpha \cdot F\left({ }_{h} \mathbf{r}-{ }_{m} \mathbf{y},{ }^{k} t-{ }^{l} s\right) .
\end{gathered}
$$

The coupled system of the $3 \cdot N$ equations with the $3 \cdot n$ unknown values $u_{l}^{m}(t), l=\overline{1,3}, m=\overline{1, n}$ can be written in a condensed form for adequately numbered load values and coefficients of the unknown

$$
\sum_{l=1}^{3} \sum_{m=1}^{n} A_{h m}^{i l} \cdot u_{l}^{m}(t)=p_{h}^{i}(t), \quad h=\overline{1, N}, \quad i=\overline{1,3} .
$$

The following denotations have been used

$$
p_{h}^{i}(t) \equiv \begin{cases}\tilde{P}_{i}\left({ }_{h} \mathbf{r}, t\right), & h=\overline{1,{ }^{1} N}, \\ \tilde{U}_{i}\left({ }_{h} \mathbf{r}, t\right), & h=\overline{{ }^{1} N+1, N}, \quad i=\overline{1,3},\end{cases}
$$




$$
\begin{gathered}
A_{h m}^{i l} \equiv\left\{\begin{array}{l}
2 \cdot \mu \cdot E_{, i j l}\left({ }_{h} \mathbf{r}-{ }_{m} \mathbf{y}\right) \cdot v_{j}\left({ }_{h} \mathbf{r}\right), \quad h=\overline{1,{ }^{1} N}, \\
\mu \cdot E_{, i l}\left({ }_{h} \mathbf{r}-{ }_{m} \mathbf{y}\right), \quad h=\overline{{ }^{1} N+1, N},
\end{array}\right. \\
i, l=\overline{1,3}, \quad m=\overline{1, n} .
\end{gathered}
$$

New temperature and displacement distributions may be defined in region $D$. For this purpose a contraction to the region $D$ of the functions $\hat{T}, \hat{u}_{i}$, defined by the formulae (34-35), will be carried out

$$
\begin{gathered}
{ }^{a} T(\mathbf{x}, t)=-\tilde{\kappa} \cdot\left[\eta \cdot \tilde{\lambda} \cdot \frac{\partial}{\partial t} \beta(D \times(0, t))+\right. \\
\left.+\frac{1}{{ }_{o} \lambda} \cdot W(D \times(0, t))\right] * F(\mathbf{x}, t)+ \\
-\tilde{\kappa} \cdot[g(D)+\eta \cdot \tilde{\lambda} \cdot \beta(D, 0)] * F(\mathbf{x}, t)+ \\
+\sum_{l=1}^{p} \sum_{m=1}^{n} \tilde{T}_{l}^{m} \cdot F\left(\mathbf{x}-{ }_{m} \mathbf{y}, t-{ }^{l} s\right),(\mathbf{x}, t) \in D \times(0, \infty), \\
{ }^{a} u_{i}(\mathbf{x}, t) \equiv \tilde{\lambda} \cdot\left[\gamma \cdot{ }^{a} T(D, t)-\beta(D, t)\right] * E_{, i}(\mathbf{z})+ \\
-\varepsilon_{i j k} \cdot \chi_{k}(D, t) * E_{, j}(\mathbf{z}) / \mu+ \\
+\sum_{m=1}^{n} \tilde{u}_{j}^{m}(t) \cdot E_{, i j}\left(\mathbf{x}-{ }_{m} \mathbf{y}\right), \\
\mathbf{x} \in D, t>0 .
\end{gathered}
$$

The expressions (46-47) create an approximate solution to the thermoelastic problem. The Duhamel-Neumann relation enables determination of the thermoelastic stress tensor

$$
\begin{gathered}
{ }^{a} \sigma_{i j}(\mathbf{x}, t)=2 \cdot \mu \cdot\left\{\tilde{\lambda} \cdot\left[\gamma \cdot{ }^{a} T(D, t)-\beta(D, t)\right] * E_{, j j}(\mathbf{x})+\right. \\
\left.+\sum_{m=1}^{n} u_{k}^{m}(t) \cdot E_{, i j k}\left(\mathbf{x}-{ }_{m} \mathbf{y}\right)\right\}+ \\
-\chi_{l}(D, t) *\left[\varepsilon_{i k l} \cdot E_{, k j}(\mathbf{x})+\varepsilon_{j k l} \cdot E_{, k i}(\mathbf{x})\right]+ \\
-\delta_{i j} \cdot \tilde{\lambda} \cdot\left[2 \cdot \mu \cdot \gamma \cdot{ }^{a} T(\mathbf{x}, t)+\lambda \cdot \beta(\mathbf{x}, t)\right] .
\end{gathered}
$$

The Fourier law (7) allows deriving the thermoelastic heat flux vector

$$
\begin{aligned}
&{ }^{a} q_{i}(\mathbf{x}, t)=-\tilde{\kappa} \cdot\left[{ }_{o} \lambda \cdot \eta \cdot \tilde{\lambda} \cdot \frac{\partial}{\partial t} \beta(D \times(0, t))+\right. \\
&+W(D \times(0, t))] * F_{, i}(\mathbf{x}, t)+ \\
&-\tilde{\kappa} \cdot{ }_{o} \lambda \cdot[g(D)+\eta \cdot \tilde{\lambda} \cdot \beta(D, 0)] * F_{, i}(\mathbf{x}, t)+ \\
&-{ }_{o} \lambda \cdot \sum_{l=1}^{p} \sum_{m=1}^{n} T_{l}^{m} \cdot F_{, i}\left(\mathbf{x}-{ }_{m} \mathbf{y}, t-{ }^{l} s\right) .
\end{aligned}
$$

\section{Acknowledgement}

The paper is supported by the Project 21-250/2007 DS.

\section{CONCLUSIONS}

The paper presents the influence of fictitious load on the solution of the quasi-static problem of coupled thermoelasticity. A coupled thermoelastic initial condition, as a logical consequence of the coupled governing equations, significantly simplified the considerations. The Helmholtz representations of the displacement and body force vectors enabled the solution to the initial-space value problem in a fictitious unbounded medium. Convolution products of fictitious load and the fundamental solutions of differential operators of both elliptic and parabolic type have been obtained as a result. Their components, called fictitious components, have been used for verification of the boundary condition in the finite time. The solution is continuously differentiable, thus enabling the determination of stress and heat flux distributions. 


\section{References}

[1] M. Schwartz, S. Green, W. A. Rutlege, Vector Analysis, Happer\&Brothers Publishers, New York (1960).

[2] W. Nowacki, Theory of Elasticity, (in Polish) Polish Scientific Publishers, Warszawa (1970).

[3] Z. Szmydt, Fourier Transform and Linear Differential Equations, (in Polish), Polish Scientific Publishers, Warszawa (1972).
[4] H. Marcinkowska, Introduction to the Theory of Partial Differential Equations, (in Polish), Polish Scientific Publishers, Warszawa (1972).

[5] J. Jankowski, A Method of Helmholtz Sources in Thermoelasticity, J. Thermal Stresses 28, 9 (2005).

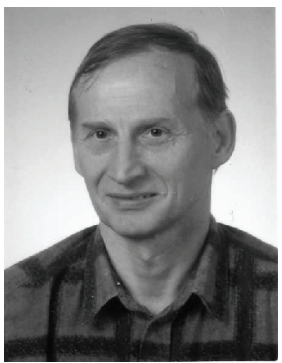

JANUSZ JANKOWSKI, born in 1947, earned his master's degree (1971) and a doctor's degree (1980) in the field of diffusion in thermoelastic medium at Poznań University of Technology. His current research interests include development of numerical methods based on some extension of the region of the body for obtaining solutions of initial-boundary value 3D problems. 